\title{
A multicenter clinical evaluation of Mycobacterium tuberculosis IgG/IgM antibody detection using the colloidal gold method
}

\author{
Y. Wang $\cdot$ B. Lu $\cdot$ J. Liu $\cdot$ T. Xiao $\cdot$ K. Wan $\cdot$ C. Guan
}

Received: 18 February 2014 / Accepted: 30 April 2014 / Published online: 10 June 2014

(C) The Author(s) 2014. This article is published with open access at Springerlink.com

\begin{abstract}
A specific immunoassay method with the colloidal gold labeling technique has been developed more and more for tuberculosis (TB) diagnosis. The aim of this multicenter clinical evaluation was to evaluate the performance of a new serological diagnostic kit (the Trustline TB IgG/IgM Rapid Test kit) for the detection of Mycobacterium tuberculosis infection in China, with the Aupu TB Ab (IgG) Colloidal Gold Test kit being used as a control. A total of 1,009 specimens were collected from three TB hospitals, including 628 patients with TB, and 219 non-TB and 162 healthy patients as
\end{abstract}

Yansen Wang and Bing Lu contributed equally to this paper.

Y. Wang $\cdot$ J. Liu $\cdot$ C. Guan $(\bowtie)$

Department of Physiology, Xiangya School of Medicine, Central

South University, Changsha, Hunan 410078, China

e-mail: guanchaxiang@csu.edu.cn

Y. Wang

e-mail: 3567556@qq.com

J. Liu

e-mail: 2549109941@qq.com

Y. Wang $\cdot$ B. Lu $\cdot$ T. Xiao $\cdot$ K. Wan $(\bowtie)$

National Institute for Communicable Disease Control and

Prevention, Chinese Center for Disease Control and Prevention, State

Key Laboratory for Infectious Disease Prevention and Control,

P.O. Box 5, Changping, Beijing 102206, China

e-mail: wankanglin@icdc.cn

B. $\mathrm{Lu}$

e-mail: lvbing2001@163.com

T. Xiao

e-mail: 983408379@qq.com

B. $\mathrm{Lu} \cdot \mathrm{K}$. Wan

Collaborative Innovation Center for Diagnosis and Treatment of

Infectious Diseases, Hangzhou 310003, China

Y. Wang

China-Japan Friendship Hospital, Yinghua East Street,

Beijing 100029, China negative controls. According to the clinical diagnostic results, the sensitivities of the Trustline kit and the Aupu kit were $61.3 \%$ and $53.7 \%$, respectively. Using the bacteriological test results as the "gold standard" to compare the results of the two kits, the sensitivity, specificity, positive predictive value (PPV), negative predictive value (NPV), and Youden index (YI) were $77.60 \%, 79.8 \%, 73.31 \%, 81.50 \%$, and 0.574 by the Trustline kit, and $67.86 \%, 88.9 \%, 83.27 \%, 77.40 \%$, and 0.568 by the Aupu kit, respectively. Further, the sensitivity of the Trustline kit and the Aupu kit for the smear staining and the bacterial culture being positive was $75.6 \%, 76.6 \%$ and $65.6 \%, 66.5 \%$, and for the negative result, it was $53.8 \%$, $50.9 \%$ and $47.5 \%, 45.0 \%$, respectively. Additionally, 35 specimens were IgM-positive by the Trustline kit; of these, 30 (4.8\%) were from patients with TB and $5(1.3 \%)$ were from individuals without TB. The results showed that the experimental test had a much higher sensitivity than the other commercial test and exhibited a good detection rate for M. tuberculosis infection. Therefore, this kit can be used in the supplementary diagnosis and screening of TB.

\section{Introduction}

It is reported that tuberculosis (TB) caused by Mycobacterium tuberculosis (M. tuberculosis) infection has become one of the major fatal infectious diseases among adults in the world [1]. China is one of the 22 countries in the world having a high TB burden and has the second highest number of active TB cases, with more than 1 million new cases of TB diagnosed each year.

Currently, patients are diagnosed with TB based mainly on clinical symptoms, X-rays, and conventional laboratory tests, such as bacterial cultures and sputum smear acid-fast staining, which exhibit much lower sensitivity. Acid-fast staining cannot be used for the diagnosis of extrapulmonary TB, such as 
Table 1 Distribution of serum samples in the three different hospitals in China

\begin{tabular}{lrrrr}
\hline \multirow{2}{*}{ Samples } & \multicolumn{2}{l}{ Samples, $n(\%)$} & \multirow{2}{*}{ Total, $n$} \\
\cline { 2 - 3 } & \multicolumn{1}{l}{ TB } & \multicolumn{1}{c}{ Non-TB } & Healthy & \\
\hline Beijing Geriatric Hospital (Beijing) & $241(72.8)$ & $36(10.9)$ & $54(16.3)$ & 331 \\
General Hospital of Huabei Oil Field Company & $188(53.7)$ & $105(30.0)$ & $57(16.3)$ & 350 \\
Cangzhou Infectious Disease Hospital (Hebei Province) & $199(60.7)$ & $78(23.8)$ & $51(15.5)$ & 328 \\
Total & $628(62.2)$ & $219(21.7)$ & $162(16.1)$ & 1,009 \\
\hline
\end{tabular}

tuberculous pleurisy, tuberculosis of lymph nodes, tuberculosis meningitis, etc., and bacterial culture is a time-consuming method that is unsuitable for rapid and early diagnosis, and it also has very low sensitivity. Therefore, researchers are interested in the development of new rapid-detection approaches, such as immunological [2] and nucleic acid [3] detection techniques.

Since the 1980s, a number of new immunoassay techniques has been developed using three labeling techniques (fluorescein, radioisotopes, and enzymes) [4]. Such methods were initially used only for immunoelectron microscopy; however, over time, these methods have been used for additional applications in passive agglutination tests, light microscopy staining, immunoblotting, immunoblot filtration assays, and immunoassays [5-7]. These techniques use chromatography membranes precoated with specific substrate(s) as the solid phase; as the sample solution moves through the membrane by means of capillary action, the analytes in the sample react with specific substrates with high affinity. The resulting immune complexes are enriched or trapped within the membrane and can be visualized with markers, such as colloidal gold. This technology does not require special equipment, the products have a long shelf life and can be stored at room temperature, the operators do not need special training, and results can be obtained within 10-20 min and observed by the naked eye, making the immune colloidal gold technique especially suitable for the majority of rural and community clinics and local hospitals, on-scene investigators, large-scale urgent testing, and general disease surveys [4, 8-12].
In this clinical trial, we evaluated the performance of the Trustline kit (produced by Beijing Genesee Biotech, Inc.) against the Aupu kit, a dot immunogold filtration assay (DIGFA) [13]. The Trustline kit, which can detect M. tuberculosis IgG/IgM antibodies, uses a colloidal gold immunochromatography method. It uses four recombinant M. tuberculosis protein antigens $(6,14,16$, and $38 \mathrm{kDa})$ simultaneously in a single-step procedure that is simple to carry out and provides rapid results.

\section{Materials and methods}

This research was approved by the Ethics Committee of the National Institute for Communicable Disease Control and Prevention, Chinese Center for Disease Control and Prevention, Beijing, China. All the patients included in this study provided signed informed consent to participate in the investigation.

In this study, the experimental kit was the Trustline TB IgG/IgM Rapid Test kit (Beijing Genesee Biotech, Inc., Beijing, China). The control kit was an M. tuberculosis IgG antibody colloidal gold kit, the Aupu TB Ab (IgG) Colloidal Gold Test kit (Shanghai Aupu Biotechnology Co., Shanghai, China), which had been approved by the China Food and Drug Administration (CFDA, authorization code 20030090).

A total of 1,009 participants' serum samples were collected from three hospitals (Table 1), among which 628 TB samples formed the case group, including 539 pulmonary TB and 89

Table 2 Demographic information for the 1,009 participants

\begin{tabular}{|c|c|c|c|c|c|c|}
\hline & & Pulmonary TB (\%) & Extrapulmonary TB (\%) & Non-TB (\%) & Healthy & Total \\
\hline Total & & $539(63.4)$ & $89(8.8)$ & 219 (21.7) & $162(16.1)$ & 1,009 \\
\hline \multirow[t]{2}{*}{ Gender } & Male & $351(60.2)$ & $56(9.6)$ & $140(24.0)$ & $86(14.8)$ & 583 \\
\hline & Female & $188(50.0)$ & $33(8.8)$ & $79(21.0)$ & $76(20.2)$ & 376 \\
\hline \multirow[t]{3}{*}{ Age } & Mean \pm SD & $45 \pm 1.83$ & $38 \pm 1.78$ & $39 \pm 2.08$ & $35 \pm 1.67$ & $43 \pm 1.96$ \\
\hline & $\leq 14$ & $7(25.0)$ & $3(10.7)$ & $6(21.4)$ & $12(42.9)$ & 28 \\
\hline & $>14$ & $532(54.2)$ & $86(8.8)$ & $213(21.7)$ & $150(15.3)$ & 981 \\
\hline
\end{tabular}


Table 3 Detection results from the 1,009 specimens using the two kits

\begin{tabular}{lllllll}
\hline \multirow{2}{*}{ Groups } & \multicolumn{2}{l}{ Trustline kit, $n(\%)$} & & \multicolumn{2}{l}{ Aupu kit, $n(\%)$} & \multirow{2}{*}{ Total } \\
\cline { 2 - 3 } & Positive & Negative & & Positive & Negative & \\
\hline TB & $385(61.3)$ & $243(38.7)$ & & $337(53.7)$ & $291(46.3)$ & 628 \\
Non-TB & $53(24.2)$ & $166(75.8)$ & & $31(14.2)$ & $188(85.8)$ & 219 \\
Healthy controls & $24(14.8)$ & $138(85.2)$ & & $11(6.8)$ & $151(93.2)$ & 162 \\
Total & 462 & 547 & & 379 & 630 & 1,009 \\
\hline
\end{tabular}

extrapulmonary TB patients, and 381 samples comprised the negative controls, including 162 medical examiners (healthy) and 219 patients with non-TB lung diseases (non-TB). The patients had an average age of $43 \pm 1.96$ years, and the gender ratio (male/female) was 1.69/1. The demographic information for the 1,009 participants is shown in Table 2. All of the 1,009 participants were negative for human immunodeficiency virus (HIV)/acquired immunodeficiency syndrome (AIDS) diagnosed with the China national diagnostic criteria and principles of management of HIV/AIDS. The antibodies against HIV in human plasma was tested with a third-generation HIV antibody enzyme-linked immunosorbent assay (ELISA) test, Vironostika HIV-1/2 Microelisa System (bioMérieux, Holland), within 24 h, according to the manufacturer's instructions [14].

Diagnoses of pulmonary TB in the study were made with the Clinical Diagnosis Standard of TB for Clinical Technology Operation (TB volumes) of the Chinese Medical Association published by the People's Medical Publishing House (PMPH; ISBN 9787117065108). Diagnoses of extrapulmonary TB were made with the Guideline of the Ministry of Health of China. In this study, we judged the final diagnosis of all patients with curative effects.

We used the two kits (Trustline and Aupu) to detect antibodies against $M$. tuberculosis in the sera samples in accordance with the respective manufacturer's instructions. We compared the kits using the sensitivity, specificity, positive predictive value (PPV), negative predictive value (NPV), Youden index (YI), and negative and positive bacteriology rates.

The bacteria from the pulmonary TB patients were detected by means of sputum smear acid-fast staining and Mycobacterium culture on Löwenstein-Jensen medium [15].

The data were analyzed using SPSS statistical software (version 16.0, SPSS Inc., Chicago, IL, USA). We used Cohen's kappa $(\mathrm{K})$ equivalence test to assess the equivalence of the two testing kits [15], and the $\mathrm{K}$-value was interpreted as follows: $\geq 0.75$, good; $<0.75$ and $\geq 0.4$, moderate; $<0.4$, poor. We used the Z-test on a series of samples to analyze whether there were differences between the test kits. Differences with $p$-values of less than 0.05 were considered to be significant.

\section{Results}

Comparison of the results of all samples tested with the two kits

For clinical diagnostic TB cases, the sensitivity of the Trustline kit $(61.3 \%, 385 / 628)$ was significant higher than that of the Aupu kit $(53.7 \%, 337 / 628)(p<0.05)$ (Table 3).

Table 4 Serum antibodies detection results from the 539 pulmonary tuberculosis (TB) cases using bacteriological methods and the two kits

\begin{tabular}{|c|c|c|c|c|c|c|c|}
\hline \multirow[t]{2}{*}{ Bacteriological methods } & \multicolumn{2}{|c|}{ Trustline (IgG and IgM), $n(\%)$} & \multicolumn{2}{|c|}{ Trustline (IgG), $n(\%)$} & \multicolumn{2}{|c|}{ Aupu (IgG), $n(\%)$} & \multirow[t]{2}{*}{ Total } \\
\hline & Positive & Negative & Positive & Negative & Positive & Negative & \\
\hline \multicolumn{8}{|l|}{ Smear staining } \\
\hline Positive & $167(75.6)$ & $54(24.4)$ & $167(75.6)$ & $54(24.4)$ & $145(65.6)$ & $76(34.4)$ & 221 \\
\hline Negative & $171(53.8)$ & $147(46.2)$ & $171(53.8)$ & $147(46.2)$ & $151(47.5)$ & $167(52.5)$ & 318 \\
\hline \multicolumn{8}{|l|}{ Bacterial culture } \\
\hline Positive & $190(76.6)$ & $58(23.4)$ & $190(76.6)$ & $58(23.4)$ & $165(66.5)$ & $83(33.5)$ & 248 \\
\hline Negative & $148(50.9)$ & $143(49.1)$ & $148(50.9)$ & $143(49.1)$ & $131(45.0)$ & $160(55.0)$ & 291 \\
\hline \multicolumn{8}{|l|}{ Total of smear + culture } \\
\hline Positive & $239(77.6)$ & $69(22.4)$ & $239(77.6)$ & $69(22.4)$ & $209(67.9)$ & $99(32.1)$ & 308 \\
\hline Negative & $99(42.9)$ & $132(57.1)$ & $96(41.6)$ & $135(58.4)$ & $87(37.7)$ & $144(62.3)$ & 231 \\
\hline
\end{tabular}

The $\chi^{2}$ test was used to analyze the $\operatorname{IgG}$ antibody positive rate of the two kits compared with smear staining and bacterial culture, respectively, $p<0.05$ 
Table 5 Comparison of the detection results by the two kits using the bacteriological test results as the "gold standard" for TB diagnosis

\begin{tabular}{lllll}
\hline Kits & Sensitivity & Specificity & PPV & NPV \\
\hline Trustline kit & $77.6 \%(239 / 308)$ & $79.8 \%(304 / 381)$ & $75.6 \%(239 / 316)$ & $81.9 \%(304 / 371)$ \\
Aupu kit & $67.9 \%(209 / 308)$ & $88.9 \%(339 / 381)$ & $83.3 \%(209 / 251)$ & $77.4 \%(339 / 438)$ \\
$p$-Value & $<0.05$ & $<0.05$ & $>0.05$ & $>0.05$ \\
\hline
\end{tabular}

NPV, negative predictive value; PPV, positive predictive value; YI, Youden index

Comparison of the results between the bacteriological methods and the two kits

Among the 628 TB cases diagnosed clinically, of the 89 extrapulmonary TB patients, the sensitivities of the Trustline kit and the Aupu kit were $52.8 \%$ (47/89) and $46.1 \%$ (42/89), respectively, and of the 539 pulmonary TB patients, the sensitivities of smear staining, bacterial culture, the Trustline kit, and the Aupu kit were $41.0 \%, 46.0 \%, 62.7 \%$, and $54.9 \%$, respectively (Table 4). The sensitivities of the Trustline kit and the Aupu kit were significantly higher than that of the bacterial methods $(p<0.05)$. The sensitivity of the Trustline kit and the Aupu kit for testing sera antibody IgG was $62.2 \%$. The sensitivity of the Trustline kit was significantly higher than that of the Aupu kit $(p<0.05)$.

Comparison of the results from the two kits using bacteriological tests as the "gold standard"

Using the bacteriological test results as the "gold standard" to compare the results of the two kits, the sensitivity, specificity, PPV, NPV, and YI were $77.60 \%, 79.8 \%, 75.6 \%, 81.9 \%$, and 0.574 by the Trustline kit, and $67.9 \%, 88.9 \%, 83.3 \%$, $77.4 \%$, and 0.568 by the Aupu kit, respectively (Table 5).

Statistical analysis of the $\operatorname{IgG}$ antibody detection results from the two kits

Using the IgG results from the Aupu kit as a reference, the positive coincidence rate of the IgG results from the Trustline

Table 6 Comparison of IgG results detected by the two kits

\begin{tabular}{llll}
\hline & \multicolumn{2}{l}{ Trustline kit } & Total \\
\cline { 2 - 3 } Aupu kit & Positive & Negative & \\
\hline Positive & 269 & 111 & 380 \\
Negative & 186 & 443 & 629 \\
Total & 455 & 554 & 1,009
\end{tabular}

The $\chi^{2}$ test was used to analyze the IgG antibody-positive rate of the Trustline kit with respect to the Aupu kit: $\chi^{2}=18.94, p=0.000, p<0.05$ kit was $70.8 \%(269 / 380)$, the negative coincidence rate was $70.4 \%$ (443/629), and the total coincidence was $70.6 \%$ (712/ 1,009) (Table 6). The equivalence test results gave a Cohen's $\mathrm{k}$-value of 0.4 , indicating moderate equivalence for $\mathrm{IgG}$ antibodies.

Comparison of the $\mathrm{IgG} / \operatorname{IgM}$ antibody detection results using the Trustline kit at the three hospitals

We compared the serum $\operatorname{IgG} / \operatorname{IgM}$ antibody results from the Trustline kit from the three hospitals. The sensitivities were $61.8 \%, 67.3 \%$, and $54.3 \%$; the specificities $61.1 \%, 83.7 \%$, and $87.3 \%$; PPVs $81.0 \%, 86.5 \%$, and $83.0 \%$; NPVs $37.4 \%$, $62.4 \%$, and $62.1 \%$; and YIs $0.23,0.51$, and 0.42 , respectively, which were all within the acceptable ranges (Table 7).

Assessment of the IgM antibody detection results measured by the Trustline kit

The results showed that, of the 381 non-TB samples, 5 cases $(1.3 \%)$ were positive, while among the 628 TB samples, 30 cases $(4.8 \%)$ were positive. Of these 30 clinically diagnosed patients who were IgM-positive, two had acute hematogenous disseminated TB, 28 cases were secondary to TB, and 26 cases $(87 \%)$ were positive for both IgG and IgM (Table 8 ).

\section{Discussion}

Several reviews have provided performance evaluations of serological tests for rapid TB diagnosis [16-18]. A comprehensive review was published by Steingart et al. [17], who used a bivariate random effects meta-analysis to prespecify subgroups in order to address heterogeneity. They also summarized test performance by analyzing papers published from January 1, 1990 to June 29, 2010 after searching multiple databases. For anti-TB IgG, the pooled sensitivities were $76 \%$ in smear-positive and $59 \%$ in smear-negative patients, and the pooled specificities were $92 \%$ and $91 \%$, respectively. Compared with ELISAs (pooled sensitivity, $60 \%$; pooled specificity, $98 \%$ ), immunochromatographic tests yielded lower pooled sensitivity (53\%) and comparable pooled specificity 
Table 7 Comparison of the $\mathrm{IgG} / \mathrm{IgM}$ antibody detection results using the Trustline kit at the three hospitals

\begin{tabular}{|c|c|c|c|c|c|c|c|c|}
\hline \multirow[t]{2}{*}{ Groups } & \multicolumn{2}{|c|}{ Beijing Geriatric Hospital } & \multicolumn{2}{|c|}{ Cangzhou Infectious Disease Hospital } & \multicolumn{2}{|c|}{ General Hospital of Huabei Oil Field Company } & \multicolumn{2}{|l|}{ Total } \\
\hline & Positive & Negative & Positive & Negative & Positive & Negative & Positive & Negative \\
\hline TB & 149 & 92 & 134 & 65 & 102 & 86 & 385 & 243 \\
\hline Controls & 35 & 55 & 21 & 108 & 21 & 141 & 77 & 304 \\
\hline Total & 184 & 147 & 155 & 173 & 123 & 227 & 462 & 547 \\
\hline
\end{tabular}

Control: non-TB +healthy; the $\chi^{2}$ test was used to analyze the $\mathrm{IgG} / \mathrm{IgM}$ antibody-positive rate tested with the Trustline kit. For the case group, $\chi^{2}=7.02$, $p=0.030, p<0.05$, and for the control group, $\chi^{2}=25.99, p=0.000, p<0.05$

(98 \%). In another study, Steingart et al. [18] used culture and clinical diagnosis methods as the reference standards. For pulmonary TB (eight test evaluations), commercial serological tests showed modest performance [diagnostic odds ratio $(\mathrm{DOR})=7.30$ ], with a pooled sensitivity of $88 \%$ and a pooled specificity of $50 \%$; for extrapulmonary TB (four test evaluations), the pooled sensitivity was less than $50 \%$ and the pooled specificity was $93 \%$. In our current study using 1,009 specimens, according to the clinical diagnostic results, the pooled sensitivities and specificities of the Trustline kit and the Aupu kit were $61.3 \%, 79.8 \%$ and $53.7 \%, 88.9 \%$, respectively $(p<0.05)$. After further analysis according to the results of smear staining and bacterial culture methods, the pooled sensitivities of the Trustline kit and the Aupu kit were $75.6 \%$, $65.6 \%$ and $76.6 \%, 66.5 \%$, repectively (Tables 4 and 5).

Analysis of the results of this clinical experiment gave a K-value of 0.4 for $\operatorname{IgG}$ detection, indicating that there was no significant difference in the overall clinical diagnostic performance of the two kits. However, further analysis showed that, using the bacteriological test results as the "gold standard" for TB diagnosis to compare the results of the two kits, both the sensitivity and specificity differed between the two kits (both $p<0.05$ ). The sensitivities of the Trustline kit and the Aupu kit for smear staining and bacterial culture negative were $53.8 \%$, $50.9 \%$ and $47.5 \%, 45.0 \%$ respectively.

China has a high rate of TB infection (up to $44.5 \%$ of the population) [1, 19], but only $5-10 \%$ of those infected exhibit

Table 8 IgM antibody detection results for the 1,009 cases tested using the Trustline kit

\begin{tabular}{llll}
\hline Case group & \multicolumn{2}{l}{ Trustline kit } & Total \\
\cline { 2 - 3 } & Positive & Negative & \\
\hline Case group & 30 & 598 & 628 \\
Control group & 5 & 376 & 381 \\
Total & 35 & 974 & 1,009 \\
\hline
\end{tabular}

The $\chi^{2}$ test was used to analyze the IgM antibody-positive rate between the case and control groups, $\chi^{2}=8.50, p=0.004, p<0.05$ symptoms of active TB, and most of the remaining patients have latent infections $[1,20]$. With the increased sensitivity of detection methods, the detection rate of latent infection will be increased, resulting in an increase in the false-positive rate and a decrease in specificity.

In the large number of clinical samples used in this study, the sensitivity of the Trustline kit was significantly better than that of the Aupu kit. In addition, the Trustline kit has the advantages of simple operation and short detection time, and can detect both anti-TB IgG and IgM antibodies simultaneously in human serum or plasma.

In summary, our data demonstrated that the Trustline TB IgG/IgM Rapid Test kit was more sensitive than the Aupu TB $\mathrm{Ab}$ (IgG) Colloidal Gold Test kit in the detection of TB antibodies in serum specimens. The Trustline TB IgG/IgM Rapid Test kit could detect both IgG and IgM in one test procedure and should be helpful for improving the detection rate of TB.

Acknowledgments We thank the staff at the three hospitals in this study (Beijing Geriatric Hospital, General Hospital of Huabei Oil Field Company, and Cangzhou Infectious Disease Hospital) for their valuable contributions to this study. We are grateful to Guizhi Sun, Baocai Yang, and Jinfeng Wang for providing the samples and patient information.

Author contributions For this study, Cha-Xiang Guan and Kanglin Wan conceived and designed the experiments. Yansen Wang, Bing Lu, Li Wan, Charles Pan Jiao Liu, and Tongyang Xiao collected the clinical samples, including the patients' information, performed the experiments, and analyzed the data. Cha-Xiang Guan and Kanglin Wan contributed reagents, materials, and analysis tools. Cha-Xiang Guan, Kanglin Wan, Yansen Wang, and Bing Lu wrote the article.

Funding This work was funded by the National Key Program of Mega Infectious Disease (2013ZX10003006-002-001 and 2013ZX10003002001), the projects of the National Natural Science Foundation of China (81170059), and the Innovation Fund for the Institution of Higher Education of Hunan Province (11 K077). The funders had no role in the study design, data collection and analysis, manuscript preparation, or decision to publish.

Competing interests The authors have read the journal's policy and declare that no competing interests exist. Patient consent for publication was obtained. 
Open AccessThis article is distributed under the terms of the Creative Commons Attribution License which permits any use, distribution, and reproduction in any medium, provided the original author(s) and the source are credited.

\section{References}

1. World Health Organization (WHO) (2011) Global tuberculosis control 2011: WHO report. WHO, Geneva

2. Chang J, Arbeláez P, Switz N et al (2012) Automated tuberculosis diagnosis using fluorescence images from a mobile microscope. Med Image Comput Comput Assist Interv 15(Pt 3):345-352

3. O'Donnell N, Corcoran D, Lucey B, Barrett A (2012) Molecularbased mycobacterial identification in a clinical laboratory setting: a comparison of two methods. Br J Biomed Sci 69:164-168

4. Chan ED, Heifets L, Iseman MD (2000) Immunologic diagnosis of tuberculosis: a review. Tuber Lung Dis 80:131-140

5. Winthrop KL, Nyendak M, Calvet $H$ et al (2008) Interferon-gamma release assays for diagnosing Mycobacterium tuberculosis infection in renal dialysis patients. Clin J Am Soc Nephrol 3:1357-1363

6. Lawn SD, Bangani N, Vogt M et al (2007) Utility of interferongamma ELISPOT assay responses in highly tuberculosis-exposed patients with advanced HIV infection in South Africa. BMC Infect Dis 7:99

7. Laboratory-based evaluation of 19 commercially available rapid diagnostic tests for tuberculosis. Diagnostics Evaluation Series No. 2. Special Programme for Research \& Training in Tropical Disease (TDR) sponsored by UNICEF/UNDP/World Bank/WHO

8. Chan E, Reves R, Belisle JT, Brennan PJ, Hahn WE (2000) Diagnosis of tuberculosis by a visually detectable immunoassay for lipoarabinomannan. Am J Resp Crit Care Med 161:1713-1719

9. Fan CM, Li W, Fan DL, Zheng XY, Ding JZ (2011) A study of double antigen sandwich colloidal gold immunochromatography rapid detection for Mycobacterium tuberculosis antibody. Zhonghua Jie He He Hu Xi Za Zhi 34:356-358

10. Daniel TM (1996) Immunodiagnosis of tuberculosis. In: Rom WN, Garay S (eds) Tuberculosis. Little, Brown \& Co., Boston, pp 223231

11. Wilkens EGL (1994) The serodiagnosis of tuberculosis. In: Davies PDO (ed) Clinical tuberculosis. Chapman \& Hall, London, pp 367379

12. Foulds J, O’Brien R (1998) New tools for the diagnosis of tuberculosis: the perspective of developing countries. Int J Tuberc Lung Dis 2:778-783

13. Hou D, Wang X, Huang Y (2001) Diagnostic value of gold standard method detecting of serum anti-TB antibody on TB. Chinese Med Factory Mine 14:413-414

14. Han $\mathrm{X}, \mathrm{Xu} \mathrm{J}$, Chu $\mathrm{Z}$ et al (2011) Screening acute HIV infections among Chinese men who have sex with men from voluntary counseling \& testing centers. PLoS One 6:e28792

15. Rieder HL, Chonde TM, Myking H; International Union Against Tuberculosis and Lung Disease et al (1998) The Public Health Service National Tuberculosis Reference Laboratory and the National Laboratory Network

16. Dinnes J, Deeks J, Kunst $\mathrm{H}$ et al (2007) A systematic review of rapid diagnostic tests for the detection of tuberculosis infection. Health Technol Assess 11:1-196

17. Steingart KR, Flores LL, Dendukuri N et al (2011) Commercial serological tests for the diagnosis of active pulmonary and extrapulmonary tuberculosis: an updated systematic review and meta-analysis. PLoS Med 8(8):e1001062

18. Steingart KR, Ramsay A, Dowdy DW, Pai M (2012) Serological tests for the diagnosis of active tuberculosis: relevance for India. Indian J Med Res 135:695-702

19. National Technic Steering Group Of The Epidemiological Sampling Survey For Tuberculosis, Duanmu H (2002) Report on fourth national epidemiological sampling survey of tuberculosis. Zhonghua Jie He He Hu Xi Za Zhi 25:3-7

20. Lin PL, Flynn JL (2010) Understanding latent tuberculosis: a moving target. J Immunol 185:15-22 